\title{
De los niños a los padres: El inventario de percepción de conductas parentales
}

\author{
César Merino Soto \& Maritza Díaz Casapía
}

Lima - Perú

Barry H. Cohen

Universidad de Nueva York

El presente estudio explora las propiedades psicométricas del Inventario de Percepción sobre los Padres (Hazzard et al., 1983), instrumento elaborado para describir, con un acercamiento global, las prácticas de crianza de los padres tal como son experimentadas y percibidas por los niños. En una muestra de 276 alumnos desde el segundo al sexto grados de primaria de un colegio no estatal, mediante el análisis de cluster se confirmó la estructura interna del inventario; por otro lado, el género de los estudiantes tanto como el grado escolar explicaron poco sobre la varianza en las diferencias entre los puntajes. Estas diferencias resultaron, en general, no significativas. Considerando las propiedades psicométricas, (1) el análisis apoyó la validez de la asumida estructura bidimensional, y (2) la confiabilidad fue aceptable.

crianza / inventario de percepción sobre los padres / evaluación / despistaje / niños / análisis de cluster

\section{Childrens and parents: The Perception of Parent Inventory}

The present study explores the psychometric properties of the Parent Perception Inventory (Hazzard et al., 1983), instrument elaborated to describe, with a global approach, the parenting practices just as they are experienced and perceived by the own children. In a sample of 276 students from the 2 do to the 6to degree of primary of a private school, the cluster analysis applied confirmed the internal structure of the inventory. On the other hand, the gender of the students as much as the school degree explained little variance in the differences in scores. These differences were, in general, not significant. On the other hand, regarding the psychometric propierties, (1) the analysis supported the validity of the assumed bi-dimensional structure; and (2) the reliability is acceptable.

parenting / Parent Perception Inventory / assessment / screening / children / cluster analysis

Correo electrónico: sikayax@yahoo.com.ar

mdiaz_casapia@hotmail.com

barry.cohen@nyu.edu

Persona 6, 2003, 135-149 
Los estilos de crianza representan un constructo ubicado en un macronivel, del que se asume que refleja las respuestas típicas de los padres a las situaciones de interacción padre-niño, las mismas que realmente definen la paternidad.

Uno de los estilos de crianza se caracteriza por una constelación de conductas parentales, que crea un persistente clima interaccional en un amplio rango de contextos y situaciones (Darling \& Steinberg, 1993). Los estilos de crianza se pueden conceptuar como algo que representa un patrón general de crian$\mathrm{za}$, caracterizado por las respuestas y técnicas específicas de los padres (Coplan, Hastings, Lagace-Seguin \& Moulton. C. E., en prensa). Estas técnicas o respuestas caracterizan a dos facetas que se han reconocido en la literatura científica: las prácticas disciplinarias y la promoción del afecto y cuidado (Maccoby \& Martin, 1983). Uno de los retos que el estudio de las prácticas de crianza ha surgido es su medición, ya sea para fines de despistaje, diagnóstico o de investigación. La investigación sobre los efectos de las prácticas de crianza en el desarrollo de desajustes ha sido consistentemente un factor de riesgo (Buchanan, 1996), como, por ejemplo, en el desarrollo de la ansiedad (Taboada, Ezpeleta \& De la Osa, 1998); así que la investigación centrada en los instrumentos que la tipifican es la regla más que la excepción.
Existen propuestas de evaluación de la crianza, como la técnica $Q$ sort de Block (1965), utilizada por Majluf (1989), y que es respondida por madres de habla hispana. Por otro lado, medidas que recogen las respuestas de los niños también han sido reportadas, como la escala de estilos de crianza de Steinberg (Lamborn, Mounts, Steinberg \& Dornbusch, 1991; Steinberg, Lamborn, Dornbusch \& Darling, 1992) que ha sido validada para su uso con adolescentes hispanos (Merino, 2003a); una medida de autonomía y compromiso de los padres percibida por los niños (Canales, 2000) y una escala de prácticas disciplinarias (Merino, 2003b).

Una opción para investigar los patrones de crianza es obtenerlo desde la propia percepción de los niños del nivel de escolaridad primaria. Un instrumento apropiado para aplicarse en situaciones de despistaje o con objetivos centrados en una caracterización de amplio rango de las formas predominantes de crianza, es el Inventario de Percepción de los Padres, IPP (Haz zard, Christiensen y Margolin, 1983). Está diseñado para ser respondido por niños de los grados primarios e inclusive con niños de cinco años. Su formato se dirige a la aplicación colectiva de alumnos sin ser mayormente intrusivo en las actividades escolares de los alumnos. El IPP se diseñó para ser un instrumento breve, de fácil administración sobre las percepciones de los 
niños sobre las conductas de sus padres. Durante las experiencias de administración, el IPP demostró ser rápidamente entendible y no provocó muchas dudas o resistencias.

Los autores sugieren los siguiente usos del IPP: (a) como instrumento al inicio y término de una terapia familiar, (b) como parte de una batería de instrumentos que exploren las conductas problemáticas de los padres, así como su incremento o decremento, y (c) como medida cualitativa de las relaciones padre-niño, comparando el grado de similaridad percibida entre los padres.

Las respuestas al IPP, en el estudio original de los autores, difirieron según el sexo del niño y del padre, mas no fueron afectados por la edad; ello sugiere que los reportes de los niños sobre las conductas de los padres deben ser analizados separadamente según el sexo del niño. La muestra en que se efectuó tal estudio fue, sin embargo, pequeña, y su generalización puede tener limitaciones (Cohen, 2001).

El presente estudio está orientado hacia la exploración de las propiedades psicométricas del IPP, para contribuir con la información que sustenten la validez y la confiabilidad de las inferencias (Tenopyr, 1977) que pueden hacerse al evaluar las prácticas de crianza.

\section{MÉTOdo}

\section{Muestra}

Este estudio exploratorio se basó en los datos de 276 estudiantes, varones y mujeres, de un colegio privado mixto en el distrito de Chorrillos. El colegio pertenece a una orden religiosa y congrega a familias cuyos ingresos y preparación educativa mayoritariamente los ubican en un nivel medio, según el informe de los administradores del colegio. Los alumnos cursaban de segundo al sexto grados de primaria, con la siguiente distribución: $26(9,4 \%)$ en segundo; $62(22,5 \%)$ en tercero; 81 $(29,3 \%)$ en cuarto; $49(17,8 \%)$ en quinto y $58(21,0 \%)$ en sexto. En la muestra $143(51,8 \%)$ fueron varones y 133 $(48,2 \%)$ mujeres. La distribución aproximada de las edades va desde los 7-8 años hasta los 11-12 años.

\section{Instrumentos}

La presente investigación utilizó el Inventario de Percepción de los Padres, un instrumento elaborado por Hazzard, Christiensen y Margolin (1983), para estudiar los patrones de crianza tal como son percibidos por los niños. La traducción se efectuó por el método backtranslation. Son 18 ítems agrupados en conductas negativas y positivas de los padres. Las 18 clases de conductas son respondidas primero para la madre y luego para el padre. El niño responde haciendo un círculo en una de las alter- 
nativas, sobre una escala de cinco puntos (0 a 4); las instrucciones y los ítems son leídos por el examinador al grupo evaluado. Se obtienen cuatro subescalas: Madre Positiva, Madre Negativa, Padre Positivo y Padre Negativo. Los ítems para las subescalas Madre Positivo y Padre Positivo están numerados con los impares $(1,3,5,7,9,11$, $13,15,17)$ y miden los siguientes comportamientos: reforzamiento positivo, confortación, tiempo para conversar, participación en toma de decisiones, tiempo para estar juntos, evaluación positiva, aceptación de independencia, asistencia y afectividad no verbal. Los ítems para las subescalas Madre Negativa y Padre Negativo son los números pares $(2,4,6,8,10,12,14,16$, 18): remoción de privilegios, crítica, órdenes, castigo físico, gritos, amenaza, tiempo fuera, regaños, ignorar.

La confiabilidad por consistencia interna (Cronbach, 1951) en el estudio original halló las siguientes estimaciones: Madre Positiva .84, Madre Negativa .87 , Padre Positivo .88 y Padre Negativo .80. Considerando la edad, la consistencia en el rango 5 a 9 años la confiabilidad interna fue de .74 a $.89, \mathrm{y}$ en el rango 10 a 13 años, de .81 a .87 .

Respecto de los estudios de validez convergente y discriminativa (Nunna1ly \& Bernstein, 1995), éstos fueron establecidos con medidas de autoconcepto del niño, encontrándose correlaciones significativas y esperadas entre las subescalas del IPP y el autoconcepto del niño. Con una medida de problemas conductuales, las puntuaciones negativas en el IPP fueron significativamente correlacionadas con las conductas problemas reportadas por los padres.

Con pruebas de rendimiento académico y desempeño cognitivo percibido, se halló relaciones no significativas en la mayoría de las subescalas del IPP, lo que comprobó el dominio teórico hacia el cual está orientado y su diferenciación con otros constructos.

Comparando los puntajes en el IPP entre familias en asistencia clínica y normales, se hallaron diferencias en las direcciones hipotetizadas, de tal modo que las familias derivadas a consulta psicológica presentaban más conductas parentales negativas, tal como fueron descritas por el IPP.

\section{Procedimiento}

Luego de obtener la autorización de la dirección del colegio, se entregó el material al responsable del servicio psicológico, quien recibió las instrucciones para la aplicación del instrumento. La psicóloga del colegio se encargó de aplicar las pruebas a los primeros grados, mientras que el autor -con el apoyo de algunos practicanteslas aplicó al resto de los grados. La administración de las pruebas se efectuó, en el contexto del aula de clase, a todos los alumnos presentes en el día de la aplicación. Se enfatizó que las respuestas no serían comentadas a sus padres. 
Con los alumnos de los primeros grados se solicitó la ayuda de la profesora de turno.

\section{Análisis}

Los análisis previos consistieron en una exploración de datos. Las pruebas de normalidad (DeCarlo, 1997) resultaron en que la distribución de las escalas no siguieron una distribución normal, observándose esto en la magnitud de la simetría distributiva. Respecto de la homogeneidad de las varianzas mantuvo niveles aceptables, a través de las submuestras. Las distorsiones en la normalidad y la heterogeneidad de varianzas, sin embargo, no fueron de gran magnitud como para afectar la aplicación de las técnicas paramétricas, aún con las diferencias significativas estadísticamente detectadas (Cohen, 2001). Aunque la aplicación de las técnicas no paramétricas es una opción coherente ante datos con desviaciones de la normalidad (Siegel, 1970), la robustez de ANOVA ante tales condiciones (Cohen, 2001) orientó su elección para probar la significancia de las diferencias entre las submuestras. Para el análisis de los efectos principales del grado y el sexo del niño, y su interacción, se crearon nuevas variables dependientes: (1) se sustrajo los puntajes positivos entre padres y madres, haciéndose lo mismo con los puntajes negativos; (2) se sustrajo el puntaje positivo entre el negativo para el padre y la madre separadamente. Un análisis de varianza de dos vías se efectuó sobre tales variables. La magnitud del efecto se informará en términos de la $d$ de Cohen y el eta (Cohen, 2001).

Se analizaron las propiedades psicométricas mediante la correlación ítem-test corregida por espuria (Thorndike, 1989) y la estimación de la consistencia interna por el coeficiente alfa (Cronbach, 1951). También, la dimensionalidad se realizó con el análisis de clusters, mediante el método de Ward (Johnson \& Wichern, 1998), que está basado en la minimización de la varianza dentro de los grupos. La distancia euclidiana sirvió para estimar la asociación entre los ítems. El algoritmo implementado proviene de Milligan y Chang (1993); esta metodología desarrollada por Milligan y sus colaboradores (Milligan \& Sokol, 1980; Milligan, 1981) incluye una medida de la bondad de ajuste de la estructura del agrupamiento, mediante la correlación punto biserial: las correlaciones más elevadas indicaron la presencia de una robusta estructura específica en los datos. Consistente con la comparación de modelos estructurales sobre los datos (Hair, Anderson, Tatham \& Black, 1999), el número de clusters hipotetizado se comparó con los datos generados aleatoriamente, ya que éstos representan el modelo nulo o aleatorio. Este método sirvió para evidenciar la validez de constructo del instrumento.

Moreno y Pérez-Gil (1993) señalan que el uso del análisis de clusters pro- 
porciona resultados similares a otras técnicas de clasificación y reducción de datos multivariados, como el análisis factorial; y es especialmente una opción preferida cuando se conoce de antemano la estructura de los datos y no se cumplen los presupuestos exigidos (por ejemplo no normalidad, datos categóricos).

\section{Resultados}

\section{Confiabilidad}

Las correlaciones ítem-test tuvieron un rango de .41 a .63 para Padre Positivo, .35 a .60 para Padre Negativo, .27 a .47 para Madre Positiva y .19 a .50 para Madre Negativa. Estas correlaciones tienden a ser, en general, similares a las encontradas por Hazzard et al. (1983) y, con dos excepciones, los ítems alcanzan óptimas correlaciones para los límites convencionalmente establecidos en el análisis de ítems (Nunnally \& Bernstein, 1995). En la tabla 1 se exponen estas estimaciones. En general, los valores de alfa se mantienen en valores que van desde niveles tan bajos como .28 (segundo grado, Madre Positiva) hasta valores elevados como .86 (cuarto grado, Padre Positivo). Se puede observar que hay una tendencia a que los valores de alfa para las escalas referidas a la madre por lo común son bajos en relación a las escalas referidas al padre.

\section{Validez}

La exploración de la agrupación de los ítems dentro de las escalas predefinidas converge con los resultados de las estimaciones de consistencia interna descritos en los siguientes párrafos. Como se esperaba, todos los ítems, excepto 1, formaron dos grupos: conductas positivas y conductas negativas por separado para Padre y Madre. El ítem que se separó de la escala Madre Negativa fue el ítem 4 ("Te dice que no estás bien, que no haces nada bien; te dice que algo no has hecho bien, te critica"), que apareció como un subcluster independiente dentro de los ítems ne-

Tabla No 1

Consistencia interna para la muestra total y sus particiones

\begin{tabular}{lccccccccc}
\hline & $\begin{array}{c}\text { Rango } \\
\text { Ritc }\end{array}$ & Total Varones & Mujeres & $2^{\circ}$ & $3^{\circ}$ & $4^{\circ}$ & $5^{\circ}$ & $6^{\circ}$ \\
\hline Escala Padre Positivo & $.41-.63$ & .82 & .80 & .84 & .75 & .78 & .86 & .87 & .67 \\
Escala Padre Negativo & $.35-.60$ & .80 & .79 & .81 & .74 & .82 & .81 & .85 & .76 \\
Escala Madre Positiva & $.27-.47$ & .73 & .73 & .71 & .50 & .66 & .73 & .80 & .79 \\
Escala Madre Negativa & $.50-.19$ & .67 & .63 & .72 & .28 & .73 & .76 & .78 & .70 \\
\hline
\end{tabular}

* correlación ítem-total corregida, para la muestra total. 
gativos; el comportamiento de este ítem no debe llamar la atención desde que su índice de discriminación es bajo $\left(r_{\text {it }}=.19\right)$. En la tabla 1 se reporta la evaluación de los resultados. Para la versión padres, hasta cinco clusters significativos $(\mathrm{p}<.0001)$ pueden ser diferenciados de aquellos generados aleatoriamente. Sin embargo, dos clusters parecen ajustarse mejor $\left(r_{p b}=\right.$ .84); además, los cambios en la fusión de los clusters es más abrupto entre los primeros y los restantes.

En la versión madres se han obtenido hasta tres clusters significativos; la bondad del ajuste de los dos primeros, sin embargo, es mayor $\left(r_{p b}=.75\right)$. De manera similar a la versión padres, el cambio en la fusión de los clusters es más marcado entre los dos primeros y el resto. Estos resultados cuantitativos convergen con lo esperado teóricamente, en que los ítems para ambas versiones se separan en conductas parentales positivas y negativas.

\section{Sesgo}

La presencia del sesgo en la medición de atributos psicológicos es una amenaza permanente para la validez de constructo (Thorndike, 1989), y afecta la calidad métrica aumentando la varianza irrelevante al constructo (Downing, 2002). Uno de los acercamientos psicométricos para estimar el sesgo a nivel de la prueba completa es la comparación de la consistencia interna (Reynolds, 1982; 2000). Nuestros resultados de la confiabilidad interna muestran una tendencia similar a los del estudio original (Hazzard et al.,

Tabla $\mathbf{N}^{\circ} 2$

Resultados del análisis de cluster para IPCP padres y madres

\begin{tabular}{lccccc}
\hline & $\begin{array}{l}N^{\circ} \text { de } \\
\text { clusters }\end{array}$ & $\begin{array}{l}\text { Valores de } \\
\text { proximidad }\end{array}$ & $\begin{array}{l}\text { Pasos de } \\
\text { fusión }\end{array}$ & R punto-biserial $\mathrm{p}$ & \\
\hline IPCP - Padres & 6 & 41.3 & -.400 & 0.487 & 0.4000 \\
& 5 & 39.3 & -1.93 & 0.577 & 0.0000 \\
& 4 & 37.8 & -1.57 & 0.612 & 0.0000 \\
& 3 & 35.3 & -2.43 & 0.753 & 0.0000 \\
& 2 & 30.7 & -4.65 & 0.848 & 0.0000 \\
IPCP - Madres & 1 & -186. & -216. & 0.000 & \\
& & & & & \\
& 4 & 34.9 & -2.01 & 0.536 & 0.0700 \\
& 3 & 34.0 & -.825 & 0.647 & 0.0000 \\
& 2 & 26.6 & -7.48 & 0.759 & 0.0000 \\
\hline
\end{tabular}


1983); así, podemos afirmar que los puntajes del instrumento en ambas versiones están mínimamente influenciados por el sesgo en la medición de los constructos crianza positiva y crianza negativa.

Como en la tabla 3, se muestra el ANOVA 2 x 5 (varón y mujer x 5 grados escolares) mostró que los efectos del grado, $\mathrm{F}(4,259)=3.3\left(\mathrm{p}>.05,0^{2}=\right.$ $.013)$, y el sexo $\mathrm{F}(1,259)=1.1(\mathrm{p}>$ $\left..05,0^{2}=.017\right)$, así como de su interacción, $\mathrm{F}(4,259)=1.16\left(\mathrm{p}>.05,0^{2}=\right.$
.018 ), sobre las conductas positivas de los padres percibidas por los niños, no fueron significativamente estadísticas. De manera similar, las diferencias entre los puntajes negativos no fueron significativas considerando el sexo, $\mathrm{F}$ (1, $259)=.00\left(\mathrm{p}>.05,0^{2}=.00\right)$; grado $\mathrm{F}$ $(4,259)=.47\left(\mathrm{p}>.05,0^{2}=.00\right) ; \mathrm{y}$ la interacción entre ellas, $\mathrm{F}(4,259)=.00$ $\left(\mathrm{p}>.05,0^{2}=.02\right)$. La información sobre la media y desviación estándar aparecen en las tablas 4 y 5 .

Tabla $N^{\circ} 3$

Resultados del ANOVA, dos vías para los puntajes de diferencia

\begin{tabular}{|c|c|c|c|c|c|c|}
\hline $\begin{array}{c}\text { Fuentes } \\
R\left(R^{2}\right)\end{array}$ & Sum cuad. & g.l. & Media Cuad. & $\mathrm{F}$ & $\mathrm{p}$ & $0^{2}$ \\
\hline \multicolumn{7}{|c|}{ Diferencias entre puntajes positivos } \\
\hline Sexo & 154.64 & 1 & 154.64 & 3.30 & .07 & .013 \\
\hline Grado & 205.85 & 4 & 51.46 & 1.10 & .35 & .017 \\
\hline Sexo $x$ grado & 217.44 & 4 & 54.36 & 1.16 & .32 & .018 \\
\hline Error & 12121.29 & 259 & 46.80 & & & \\
\hline Total & 12693.0 & 269 & & & & \\
\hline \multicolumn{7}{|c|}{ Diferencias entre puntajes negativos } \\
\hline Sexo & .40 & 1 & .40 & .008 & .92 & .000 \\
\hline Grado & 90.62 & 4 & 22.65 & .47 & .75 & .007 \\
\hline Sexo $\times$ grado & 319.91 & 4 & 79.97 & 1.67 & .15 & .025 \\
\hline Error & 12361.23 & 259 & 47.72 & & & \\
\hline Total & 14835.0 & 269 & .40 & & & \\
\hline \multicolumn{7}{|c|}{ Diferencias entre puntajes sobre la madre } \\
\hline Sexo & 540.72 & 1 & 540.72 & 4.83 & .02 & .018 \\
\hline Grado & 958.90 & 4 & 239.72 & 2.14 & .07 & .031 \\
\hline Sexo $x$ grado & 85.17 & 4 & 21.29 & .190 & .94 & .003 \\
\hline Error & 29630.35 & 265 & 111.81 & & & \\
\hline Total & 100212.0 & 275 & & & & \\
\hline \multicolumn{7}{|c|}{ Diferencias entre puntajes sobre el padre } \\
\hline Sexo & 208.934 & 1 & 208.934 & 1.71 & .19 & .007 \\
\hline Grado & 875.450 & 4 & 218.863 & 1.79 & .12 & .027 \\
\hline Sexo $x$ grado & 531.112 & 4 & 132.778 & 1.09 & .36 & .017 \\
\hline Error & 31511.362 & 259 & 121.665 & & & \\
\hline Total & 122840.000 & 269 & & & & \\
\hline
\end{tabular}


Tabla $N^{\circ} 4$

Media y desviaciones estándar por grado y sexo para las escalas Padre Positivo y Padre Negativo

\begin{tabular}{lccccccr}
\hline \multirow{2}{*}{ Grado } & \multicolumn{2}{c}{ Total } & \multicolumn{2}{c}{ Masculino } & \multicolumn{2}{c}{ Femenino } & D \\
& & Media & D. E. & Media & D. E. & Media & D. E. \\
\hline Escala Padre Positivo & & & & & & & \\
$\quad$ Segundo & 26.5 & 7.3 & 28.3 & 5.5 & 24.5 & 8.7 & 0.53 \\
$\quad$ Tercero & 26.7 & 6.7 & 26.6 & 6.7 & 26.8 & 6.7 & -0.03 \\
$\quad$ Cuarto & 24.2 & 8.0 & 24.9 & 6.8 & 23.4 & 9.3 & 0.19 \\
Quinto & 24.3 & 8.3 & 22.6 & 8.7 & 26.2 & 7.6 & -0.44 \\
$\quad$ Sexto & 27.2 & 5.0 & 27.1 & 5.2 & 27.2 & 4.9 & -0.02 \\
$\quad$ Total & 25.6 & 7.3 & 25.6 & 6.9 & 25.6 & 7.6 & 0.00 \\
Escala Padre Negativo & & & & & & & \\
$\quad$ Segundo & 6.6 & 6.6 & 6.1 & 4.7 & 7.3 & 8.7 & -0.18 \\
$\quad$ Tercero & 6.8 & 6.6 & 7.7 & 6.5 & 5.6 & 6.6 & 0.32 \\
$\quad$ Cuarto & 7.1 & 6.3 & 8.6 & 5.9 & 5.5 & 6.4 & 0.50 \\
$\quad$ Quinto & 9.1 & 7.3 & 10.6 & 8.5 & 7.3 & 5.2 & 0.46 \\
$\quad$ Sexto & 7.3 & 4.9 & 8.9 & 5.2 & 6.0 & 4.2 & 0.62 \\
$\quad$ Total & 7.4 & 6.3 & 8.6 & 6.4 & 6.1 & 6.0 & 0.40 \\
\hline
\end{tabular}

Tabla $N^{\circ} 5$

Media y desviaciones estándar por grado y sexo para las escalas Madre Positiva y Padre Negativa

\begin{tabular}{|c|c|c|c|c|c|c|c|}
\hline \multirow[b]{2}{*}{ Grado } & \multicolumn{2}{|c|}{ Total } & \multicolumn{2}{|c|}{ Masculino } & \multicolumn{2}{|c|}{ Femenino } & \multirow[t]{2}{*}{$\mathrm{D}$} \\
\hline & Media & D. E. & Media & D. E. & Media & D. E. & \\
\hline \multicolumn{8}{|c|}{ Escala Madre Positiva } \\
\hline Segundo & 25.1 & 5.8 & 24.1 & 6.5 & 26.3 & 4.8 & -0.38 \\
\hline Tercero & 26.1 & 6.0 & 25.7 & 6.9 & 26.5 & 4.4 & -0.13 \\
\hline Cuarto & 24.4 & 6.1 & 24.7 & 6.0 & 24.2 & 6.3 & 0.08 \\
\hline Quinto & 26.0 & 6.6 & 24.8 & 7.1 & 27.3 & 5.8 & -0.38 \\
\hline Sexto & 28.1 & 5.8 & 27.3 & 5.7 & 28.7 & 5.9 & -0.24 \\
\hline Total & 25.9 & 6.2 & 25.4 & 6.4 & 26.5 & 5.8 & -0.18 \\
\hline \multicolumn{8}{|c|}{ Escala Madre Negativa } \\
\hline Segundo & 11.6 & 8.3 & 13.1 & 9.5 & 9.8 & 6.8 & 0.39 \\
\hline Tercero & 8.7 & 6.2 & 9.1 & 7.4 & 8.1 & 4.2 & 0.16 \\
\hline Cuarto & 10.0 & 6.5 & 11.2 & 6.3 & 8.7 & 6.5 & 0.39 \\
\hline Quinto & 11.8 & 6.9 & 12.2 & 7.7 & 11.2 & 6.0 & 0.14 \\
\hline Sexto & 9.4 & 5.2 & 9.8 & 5.0 & 9.0 & 5.4 & 0.15 \\
\hline Total & 10.0 & 6.5 & 10.8 & 7.0 & 9.2 & 5.8 & 0.25 \\
\hline
\end{tabular}


Respecto de los efectos de sexo y grado sobre la percepción del padre, no se han hallado resultados significativos; en cambio, únicamente las diferencias de género en los niños han impactado en las conductas maternales percibidas. Las niñas tienden a percibir hacia ellas un mayor número de conductas positivas de sus madres que los niños, $\mathrm{t}(274)=2.07, \mathrm{p}<.05$. Estas diferencias, sin embargo, son moderadas $(d$ $=0.38)$.

\section{Discusión}

Convergiendo con los resultados del estudio de Hazzard, Christensen y Margolin (1983), el IPP muestra que es un instrumento potencialmente apropiado para la exploración de las percepciones de los niños sobre las conductas parentales en el contexto de la crianza, definidas por dos clusters de patrones: conductas positivas y conductas negativas. Sobre las diferencias de la edad (estimada por los grados escolares) y el sexo, no se ha detectado el patrón que Hazzard et al. (1983) hallaron. Ellos encontraron que un efecto principal (sexo del niño) y de interacción (sexo del niño x sexo del padre) fueron significativos, basados en una muestra de 75 niños. En nuestro estudio los efectos no se confirmaron consistentemente, aunque sí encontramos los efectos del sexo del niño sobre la percepción de la madre. Es decir, que las niñas tienden a percibir como más positivas a sus madres que como lo ha- cen los niños; estas diferencias son, sin embargo, marginalmente moderadas.

Los niveles de confiabilidad han sido aceptables, aunque tienden a ser en algunos casos bajos en comparación con lo reportado en el estudio de Hazzard et al. (1983). Esta variabilidad parece desprenderse de errores de medición contextualmente presentes en la aplicación de la prueba, mas no del posible sesgo en la prueba.

Un hallazgo interesante respecto de las estimaciones de la confiabilidad es que los coeficientes alfa tienden a variar entre los grados escolares; o, lo que es similar, varían respecto de la edad. El pico más alto se encuentra en los grados cuarto y quinto, pero en los primeros grados y en el último los niveles de confiabilidad tienden a ser marginales, o muy bajo para los niños de segundo grado. El análisis de la consistencia interna da pruebas de la adecuación de los ítems a sus respectivas escalas, con excepción de un ítem (crítica) que evalúa un comportamiento negativo de la madre. El apoyo concerniente a la dimensionalidad de los ítems se encontró también con la exploración mediante el análisis jerárquico de clusters, que resultó en las agrupaciones esperadas de los ítems. La homogeneidad interna de los dos clusters nos puede sugerir que resultados equivalentes podemos hallar con otras técnicas de reducción de datos, como el análisis factorial (Moreno y Pérez-Gil, 1993), los cuales exigen el 
cumplimiento de varios presupuestos paramétricos (Johnson \& Wichern, 1998; Hair et al., 1999).

Al parecer, la varianza de error tiende a disminuir a través de una tendencia curvilínea con el grado escolar; tal es así que en los grados cuarto y quinto los valores del coeficiente alfa alcanzan su máximo nivel, pero tiende a disminuir nuevamente en sexto grado. Esta relación se asemeja a una asociación curvilínea y desde este hallazgo nos conduce a suponer que cuando se aplique el IPP los grupos que más confiablemente proporcionarán resultados utilizables serán los grados superiores (aun con aquel relativamente bajo nivel de confiabilidad) y en menor medida los grados inferiores. Asumimos que tal grado de error asociado a la aplicación del IPP en los grados inferiores es consecuencia de los procedimientos de aplicación ejecutados probablemente por los profesores, quienes no están familiarizados con las reglas de aplicación estándar de pruebas colectivas. A la situación anterior se agrega la poca experiencia de los niños más pequeños para responder a pruebas con formatos como los del IPP. Si extrapolamos estas conclusiones hacia grupos de niños procedentes de familias y contextos de bajo nivel sociocultural, las dificultades para responder apropiadamente aumentarían debido a que las habilidades de comprensión verbal no estarían óptimamente desarrolladas e introduciría factores de error en la aplicación (Merino \& Kenny, 2003).
Adicionalmente, los niveles relativamente bajos de confiabilidad interna han tendido a ubicarse en la percepción de las conductas de crianza de la madre. De esta manera, los coeficientes alfa promedio para Padre Positivo y Padre Negativo están alrededor de $.80(.79 \mathrm{y}$ .80 , respectivamente), mientras que para Madre Positiva y Madre Negativa es .60 y .6 respectivamente. Considerando la muestra total y clasificada por sexo, esta disminución se evidenció más en la percepción de conductas negativas de la madre, lo que señala un probable efecto de la ambivalencia o confusión que pueden sentir los niños(as) ante las prácticas disciplinarias de la madre, percibiéndolas como amenazadoras y punitivas, o como agentes benignos de control. Esto puede estar influenciado por el impacto del contexto en que se desarrolla la crianza (Darling \& Steinberg, 1993), aunque merece adicional exploración.

En el estudio original (Hazzard, Christensen \& Margolin, 1983), el IPP se administró aun a niños de cinco años, aunque no detallan si la aplicación fue individual o colectiva. De los resultados actuales y de la experiencia de aplicaciones anteriores a muestras de menores (Spielberger, 1973; Papay \& Spielberger, 1986), se desprende la necesidad de aplicar el IPP en sesiones con pequeños grupos de niños o individualmente, especialmente a niños desde cinco años (inicial) hasta segundo año de primaria. 
Las debilidades del presente estudio son la ausencia de trabajos de validación convergente, la singularidad de la muestra y la preparación de los profesores. En este sentido, no pudieron efectuarse correlaciones con criterios externos relevantes para apoyar la validez del constructo. En segundo lugar, la muestra proviene de un solo colegio de gestión no estatal y de una línea educativa de tipo religioso; las características socioeconómicas de los niños evaluados y la selección no aleatoria de esta muestra limitan la generalización de los resultados a muestras de niños de estratos bajo o medio-bajo, e incluso a poblaciones similares a la muestra estudiada. Finalmente, consideramos que la preparación de los profesores para la administración del IPP no alcanzó niveles óptimos y que debería enfatizarse tal capacitación si es que los docentes aplicaran el IPP como parte de un estudio o de evaluaciones de despistaje masivo sobre las características generales de la crianza.

Aprovechando las limitaciones como elementos para la agenda de investigaciones, estudios adicionales de validación y de generalización de los resultados a muestras de niños claramente pertenecientes a niveles socioeconómicos bajo y medio-bajo darán más soporte al uso del IPP. Otros estudios de validación incluirían la comparación de muestras clínicas y no clínicas, que revelaría posiblemente patrones de crianza diferentes; o niños de zonas periurbanas y urbanas, en cuyos ambientes se han encontrado diferencias en los estilos de crianza (Majluf, 1989) y en la socialización (Schade \& Rojas, 1989). Además, la necesidad de instrumentos como el IPP se hace más notoria a la luz de la creciente urgencia por prevenir la incidencia del maltrato infantil intrafamiliar. Como herramienta para el despistaje masivo en las actividades de prevención primaria en los colegios y centros de atención infantil, materiales como el Inventario de Percepción de los Padres facilitan el análisis de los resultados en el nivel de los puntajes totales y de los ítems, proporcionando información para explorar más profundamente los patrones que podrían ser sospechosos de disfunciones en la crianza y/o de crónicos episodios de maltrato. Aun con el adecuado balance entre costo-beneficio que presentan los métodos de autoreporte, como el IPP, su uso en una estrategia multimétodo será más apropiado en la recolección de datos para la investigación y la práctica clínica (Shelton, Frick \& Wootton, 1996).

Uno de los aspectos resaltantes de nuestros hallazgos proviene de la metodología de análisis. Aplicamos un método de análisis de cluster con medidas estadísticas de la bondad del ajuste para el número de clusters, que es consistente con la metodología de investigaciones multivariadas (Hair et al., 1999); pero debido al análisis de clusters tiende a ser bastante variable 
(Aldenderfer \& Blashfield, 1984), y la validación en otra muestra será el elemento clave de posteriores estudios. Justamente, los autores del presente artículo reportarán la extensión de la validación de este instrumento en una muestra de niños de nivel socioeconómico bajo y con una modificación del método de respuesta.

\section{REFERENCIAS}

Block, J.H. (1965). The child rearing practices report. Berkeley: Institute of $\mathrm{Hu}-$ man Development, Universidad de California.

Brown, F.G. (1980). Principios de la medición en psicología y educación. México D.F.: El Manual Moderno.

Buchanan, A. (1996). Cycles of child maltreatment. Chinchester, England: John Wiley.

Canales, N. (2000). Relación entre la percepción del estilo de crianza con el rendimiento académico mediado por la percepción de control académico y la percepción de competencia académica. Tesis para optar el título profesional en enfermería, Universidad Peruana Cayetano Heredia.

Cohen, B.H. (2001). Explaining psychological statistics (2 ${ }^{\mathrm{a}}$. ed.). New York: John Wiley \& Sons.

Coplan, R.J., Hastings, P.D., LagaceSeguin, D.G., \& Moulton, C.E. (en prensa). Authoritative and Authoritarian Mothers' Parenting Goals, Attributions, and Emotions Across Different Childrearing Contexts. Parenting: Science and Practice.

Cronbach, L.J. (1951). Coefficient alpha and the internal structure of test. Psychometrika, 6, 297-334.

Darling, N., \& Steinberg, L. (1993). Parenting style as context: An integrative model. Psychological Bulletin, 113 (3), 487-496.

DeCarlo, L.T. (1997). On the meaning and use of kurtosis. Psychological Methods, 2 (3), 292-307. 
Hair, J.F., Jr., Anderson, R.E., Tatham, R.L. \& Black, W.C. (1999). Análisis multivariante (5a. ed.). Madrid: Prentice Hall Iberia.

Hazzard, A., Christiensen, A. \& Margolin, G. (1983). Children's perceptions of parental behaviors. Jornal of Abnormal Child Psychology, 11(1), 49-60.

Johnson, R.A. \& Wichern, D.W. (1998). Applied multivariate statistical analysis. Upper Saddle River: Prentice Hall.

Lamborn, S.D., Mounts, N.S., Steinberg, L. \& Dornbusch, S.M. (1991). Patterns of competence and adjustment among adolescents from authoritative, authoritarian, indulgent, and neglectful families. Child Development, 62, 1049-1065.

Maccoby, E.E. \& Martin, J.A. (1983). Socialization in the context of the family: Parent-child interaction. En P.H. Mussen (general ed.) and E.M. Hetherington (series ed.), Manual of child psychology. Vol. 2, Social development, New York: John Wiley.

Majluf, A. (1989). Prácticas de crianza en madres de estratos socioeconómicos medio y bajo en Lima. Revista de Psicología, 7 (2), 151-161.

Merino, C. (2003a). Análisis factorial confirmatorio y confiabilidad de la Escala de Estilos de Crianza de L. Steinberg. Tesis para la titulación en psicología. Universidad Inca Garcilaso de la Vega, Facultad de Psicología y Ciencias Sociales.

Merino, C. (2003b). Desarrollo y validación inicial de una escala de prácticas disciplinarias para niños. Documento no publicado, Defensoría Municipal del Niño y del Adolescente, Lima, Chorrillos.
Merino, C. \& Kenny, D. (2003). Revisión de algunos conceptos y recomendaciones en la medición psicopedagógica. Actualidad Psicodepagógica. En revisión.

Milligan, G.W. (1981). A Monte Carlo Study of Thirty Internal Criterion Measures for Cluster Analysis. Psychometrika, 46, 187-199.

Milligan, G.W. \& Sokol, L. (1980). A Two Stage Clustering Algorithm with Robust Recovery Characteristics. Educational and Psychological Measurement, 40, 755-759.

Milligan, G.W. \& Chang, R. (1993) HCINFLY 2.0: Generalized agglomerative hierarchical clustering algorithms with internal influence measurement (Computer Program). Ohio State University.

Moreno, R. \& Pérez-Gil, J.A. (1993). On equivalence of factor analysis, linear discriminant analysis and cluster analysis. En R. Steyer, K.F. Wender \& K.F. Widaman (eds.), Psychometric Methodology. Proceedings of the 7th European Meeting of the Psychometric Sociaty in Trier (343-348). Stuttgart and New York: Gustav Fischer Verlag.

Nunnally, J.C., \& Bernstein, I.J. (1995). Teoría psicométrica (3a. ed.). México D.F.: McGraw-Hill.

Papay, J.P., \& Spielberger, C.D. (1986). Assessment of anxiety and achievement in kindergarten and first- and second grade children. Journal of Abnormal Child Psychology, 14 (2), 279-286.

Reynolds, C.R. (2000). Methods for detecing and evaluating cultural bias in neuropsychological test. In Fletcher- 
Janzen, L.S. Strickland \& Reynolds, C.R., Handbook of Cross-Cultural Neuropsychology (249-285). New York: Kluwer Academic/Plenum Publishers.

Reynolds, C.R. (1982). Construct and predictive bias. En R.A. Berk, Handbook of methods for detecting test bias (pp. 199-227). Baltimore, MD: Johns Hopkins University Press.

Schade, B. \& Rojas, C. (1989). Niños en extrema pobreza,... ¿Socialización deficitaria?: Resultado de un estudio empírico en un pueblo joven de Lima. Revista de Psicología, 7 (2), 139-150.

Shelton, K.K., Frick, P.J., \& Wootton, J. (1996). Assessment of parenting practices in families of elementary schoolage children. Journal of Clinical Child Psychology, 25 (3), 317-329.

Siegel, S. (1970). Estadística no paramétrica. México, D.F.: Trillas.

Spielberger, C.D. (1973). State-Trait ansiety Inventory for Children: Preliminary Manual. Palo Alto: Consulting Psychologists Press.

Steinberg, L., Lamborn, S.D., Dornbusch S.M. \& Darling, N. (1992). Impact of parenting on adolescent achievement: Authoritative parenting, school involvement, and encouragement to succeed. Child Development, 63, 1266-1281.

Taboada, A., Ezpeleta, L. \& De la Osa, C. (1998) Trastornos por ansiedad en la infancia y adolescencia: factores de riesgo. Ansiedad y Estrés, 4 (1), 1-16.

Tenopyr, M.L. (1977) Content-construct confusion. Personnel Psychology, 30, 47-54.

Thorndike, R.L. (1989). Psicometría aplicada. México D.F.: Limusa. 